\title{
Optical and digital microscopic imaging techniques and applications in pathology
}

\author{
Xiaodong Chen ${ }^{\mathrm{a}, \mathrm{c}}$, Bin Zheng ${ }^{\mathrm{b}}$ and Hong $\mathrm{Liu}^{\mathrm{c}, *}$ \\ ${ }^{a}$ College of Precision Instruments, Tianjing University, Tianjing, China \\ ${ }^{\mathrm{b}}$ Department of Radiology, University of Pittsburgh, Pittsburgh, PA, USA \\ ${ }^{\mathrm{c}}$ Center for Bioengineering and School of Electrical and Computer Engineering, \\ University of Oklahoma, Norman, OK, USA
}

\begin{abstract}
The conventional optical microscope has been the primary tool in assisting pathological examinations. The modern digital pathology combines the power of microscopy, electronic detection, and computerized analysis. It enables cellular-, molecular-, and genetic-imaging at high efficiency and accuracy to facilitate clinical screening and diagnosis. This paper first reviews the fundamental concepts of microscopic imaging and introduces the technical features and associated clinical applications of optical microscopes, electron microscopes, scanning tunnel microscopes, and fluorescence microscopes. The interface of microscopy with digital image acquisition methods is discussed. The recent developments and future perspectives of contemporary microscopic imaging techniques such as three-dimensional and in vivo imaging are analyzed for their clinical potentials.
\end{abstract}

\section{History of microscope development}

More than 2000 years ago, around one century B.C., people already had discovered that one could view an enlarged image of a small object by using a transparent "lens" with a spherical shape. Although a single convex lens can magnify an object by more than ten times, it is quite insufficient for people clearly to observe the details of many small objectives [1]. Near the end of 16th century, Dutch eyeglass dealer, Janssen, and his son inserted several lenses into a cylinder and found that an object was enlarged significantly if viewed through this assembled cylinder. This was the first prototype of a modern microscope and telescope. Based on this accidental discovery, Janssen designed and assembled the first compound microscope that included two convex lenses. The basic concept of a compound microscope is that an object can be enlarged sequentially by two convex lenses.

\footnotetext{
*Corresponding author: E-mail: Liu@ ou.edu.
}

While observing the soft-wood specimen using a microscope in 1665 , the British scientist, Robert Hooke, surprisingly discovered that the specimen depicted a series of unique "elements." Hooke named them "cells." Years later, a new microscope designed and assembled by a Dutch scientist, Anthony van Leeuwenhoek, provided much higher magnification power, which enabled people to observe substantially more details of cells. Although it exceeded all precedent microscopes, it was not another compound microscope and actually used only one convex lens. However, due to Leeuwenhoek's excellent manufacturing skill, this specially manufactured single convex lens achieved more than 300 times of magnification power.

In the following two centuries, the magnification power, as well as the image quality of the compound microscope, was improved substantially. This occurred, in particular, after the discovery and use of the new lens assemblies were able to eliminate or minimize the chromatic and other optical aberrations. Compared to 
the microscopes developed in 19th century, the conventional optical microscopes used to date have no substantial difference or improvement, because the optical microscopes have reached their maximum limitation in the spatial resolution.

Due to the large range or fluctuation of optical wavelength, it is impossible for any optical instruments to create a perfect image of a natural object, even if all shape defects in manufacturing optical lens were eliminated. Due to the unavoidable diffraction of optical wave passing through the microscopic lens, any point depicted on an object is no longer the point in the image plane but a diffraction spot. If two diffraction spots are too close together, the two points are not distinguishable. Under such circumstance, increasing magnification power of the microscopic objective lenses cannot further increase the spatial resolution of the microscopes. For the microscopes using the light sources within the range of visible optical wavelength, their spatial resolution is limited to $0.2 \mu \mathrm{m}$. Thus, any structures smaller than $0.2 \mu \mathrm{m}$ cannot be distinguished using this type of microscope.

One approach to increasing spatial resolution of microscopes is to reduce optical wavelength or use electron beam to replace visible light source. According to the de Broglie theory of matter wave, moving electrons is similar in nature to optical wave fluctuation. The faster that electrons move, the shorter the "wavelength" of the released energy. If the electrons can be accelerated sufficiently and the released energy can be converged, the moving electron beam also can enlarge the objects. Based on this concept, German engineers, Max Knoll and Ernst Ruska, assembled the first transmission electron microscope (TEM) in the world in 1938 [2]. British engineer, Charles Oatley, manufactured the first scanning electron microscope (SEM) in 1952 [3]. Electron microscopy was one of the most important inventions in 20th century. Because electrons can be accelerated to very high speed, the spatial resolution of electron microscopes reaches up to $0.3 \mathrm{~nm}$. As a result, many invisible materials (i.e., virus) under the visible light become "visible" under the electron microscope.

In 1983, two scientists in IBM laboratory, Gerd Binning and Heinrich Rohrer, invented the scanning tunnel electron microscope (STM) [4]. This advanced microscope was built using totally different concepts than those used by the conventional microscopes. STM works based on the so called "tunnel effect". STM has no lens; rather, it uses a probe. Once the voltage is added between the probe and the observed object, the tunnel effect occurs if the distance between the probe and the surface of the observed objects is sufficiently small (i.e., measured in nanometers). When the electrons pass through the tiny space between the probe and the object, weak electronic current is generated. If the distance between the probe and the object varies, the strength of the current varies as well. Hence, the threedimensional shape of the object can be detected as one measures the electronic current change. The spatial resolution of the STM can reach the level of a single atom.

\section{The basic concept of microscopic imaging}

Although many different types of microscopes have been developed, the basic imaging concept and structures can be simply illustrated in Fig. 1. The optical system of a microscope mainly includes an objective lens and eyepieces. The purpose of an objective lens is to magnify an object so that it can be clearly observed by the user. During the observation, the specimen is placed near the focal plane of the objective lens in the object space, and a magnified real image of specimen is first created on the intermediate plane. The intermediate plane is located on the focal plane of the eyepiece, thus the eyepiece is working as a magnifier to further magnify the image projected on the intermediate image plane. Finally, a magnified, virtual, inverted image is provided for the observer.

For a well designed microscope, the spatial resolution is mainly determined by the objective lens. Although an eyepiece can also magnify the image, it cannot improve the resolving power of the microscopes. The spatial resolution of an optical microscope is given by the Rayleigh equation as follows [5]:

$$
\Delta r_{0}=0.62 \lambda / n \sin \alpha
$$

where $\Delta r_{0}$ is the minimum resolvable distance, $\lambda$ is the wavelength of the light source; $n$ is the refractive index between the lens and the object, $\alpha$ is the half-angle aperture - the half inclination of the lens to the objective points, and $n \times \sin (\alpha)$ is the numerical aperture (NA) of the objective lens.

In the case of digital microscopic systems, the image magnified by the objective lens is directly acquired (or through a relay lens) by electronic detectors such as CCD or CMOS detectors [6, 7]. The electronic detectors are selected so that their pixel pitch is smaller 


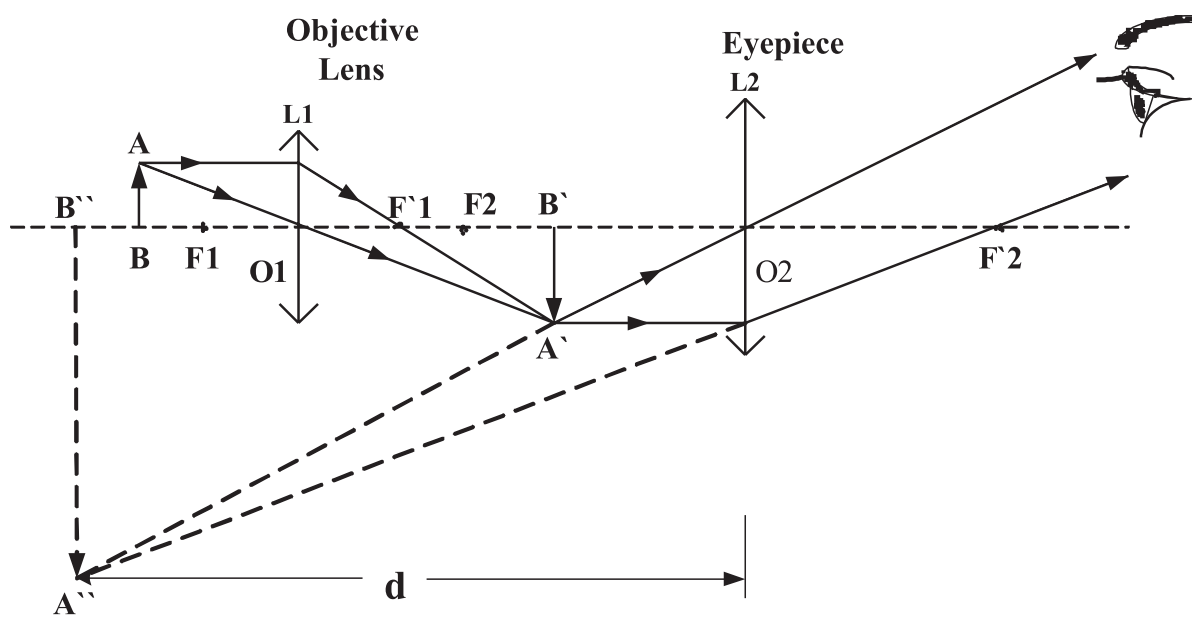

Fig. 1. The optical principle of microscope imaging.

than the magnified minimum resolvable distance determined by the objective lens. So that the resolving power of the microscope is not degraded by the digital detectors.

Based on the above equation, and considering the following practical limitations, (1) the use of visible light with the wavelength between $390 \mathrm{~nm}$ and $760 \mathrm{~nm}$, (2) the maximally reachable aperture with the halfangle of 70-75 degree, and (3) the requirement of using immersion methods with water or oil to increase of refractive index, the resolution of a conventional optical microscope cannot exceed $200 \mathrm{~nm}$.

Since the wavelength of electronic wave (beam) is much shorter than the visible light waves in several orders of magnitude, the resolution of a microscope using electronic beam in theory can reach approximately $0.3 \mathrm{~nm}$. For this reason, electron microscopy $[8,9]$ is developed based on the principles of electronic optics, which can image the fine structure of objects at very high magnification power by using the electron beam and electron lens instead of optical lens. The transmission electron microscope (TEM) is the primary representation of the electron microscopes. The TEM is so named because the electron beam first penetrates the sample and then is magnified by the electronic imaging lens to produce the images. Its optic path is similar to the conventional optical microscope (as shown in Fig. 2). After passing through convergent lens, the parallel electron beam reaches the sample. Once the electron beam passes through the sample, it carries the information related to the sample characteristics. An electronic image is first generated once

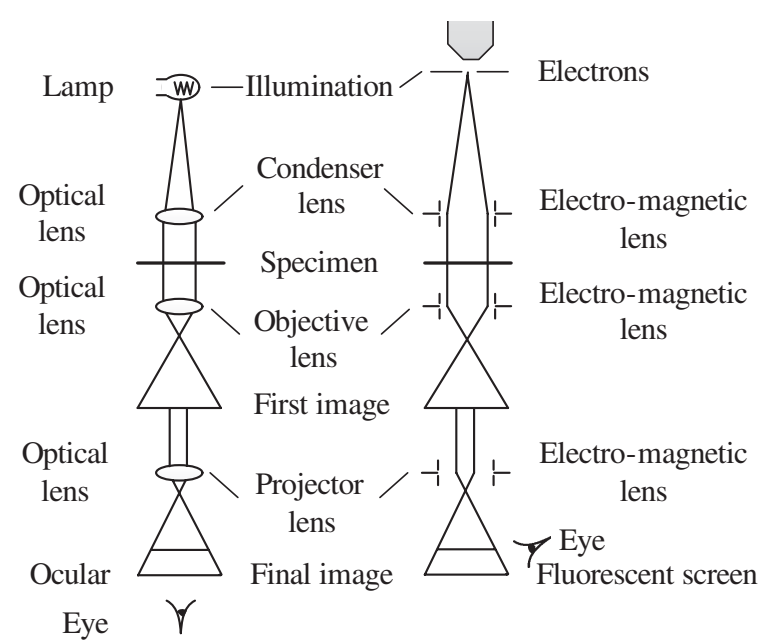

Fig. 2. Comparison of the optical microscopic and electron microscopic systems.

the electron beam passes though the objective lens and contrast aperture. After the electronic image is magnified again by the intermediate lens and projection lens, the final electron image is displayed in the electronic monitor screen. The contrast of the electron image is determined by the scattering level of electron beam once it interacts with the atoms in the sample. The electron beam also scatters less in the thinner samples or some parts with lower density. Thus, more electrons pass through lens aperture and result in the brighter image. On the other hand, thicker samples or denser parts appear darker in the image. If the sample is too 
thick or too dense, the image contrast is decreased substantially.

\section{The microscopes used in biomedical fields}

Although a large number of different types of microscopes have been developed and applied in different applications, the microscopes actually can be divided into three categories: (1) optical microscopes, (2) electron microscopes, and (3) scanning tunnel electron microscopes based on the microscope development history.

\subsection{Optical microscopes}

The simplified optical wave path of a conventional optical microscope is illustrated in Fig. 3. The modern optical microscope is able to magnify an object by 1500 times with the $0.2 \mu \mathrm{m}$ limit in spatial resolution. The optical microscopes can be divided into many different types using a variety of criteria. For example, based on a lighting method, there are the transmission and reflection types of microscopes. In a transmission microscope, the light passes through transparent objects. In a refection microscope, the light source installed on the top of the microscopic lens illuminates the non-transparent objects, and the reflected

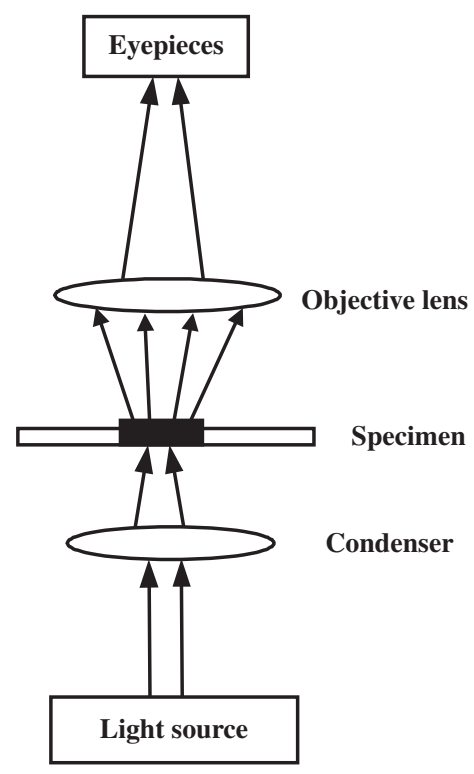

Fig. 3. Illustration of optical path of the microscope. light is collected by the lens. The microscopes can also be differentiated based on the observation methods, including bright field microscopes, dark field microscopes, phase difference microscopes, polarized light microscopes, interference microscopes, and fluorescent microscopes [5, 10-13]. Each microscope can use either the transmission or reflection approach. The bright field microscopes are the most popular and widely used of all microscopes. Using this type of microscope, the transmission (or absorption) ratio and reflection ratio of some observed objects vary according to the change of working environments. The amplitude of these objects varies with the change in lighting intensity. The colorless transparent objects are visible only when the phase of illuminated light changes. Because the bright field microscopes cannot change light phase, the colorless transparent specimens are invisible when using this type of microscope.

\subsection{Electron microscopes}

The resolution of the electron microscopes typically is represented by the small distance of two adjacent and distinguishable points. In the 1970s, transmission electron microscopes were able to reach the resolution of $0.3 \mathrm{~nm}$, while the resolution limit of the human eye is around $0.1 \mathrm{~mm}$. Compared to the optical microscopes that have the maximum magnification power of a few thousand times, the modern electron microscopes can increase the maximum magnification power to more than 300 million times. Using electron microscopes, one can directly observe some biological structures and/or the atomic structures of cells. Despite having superior spatial resolution over the optical microscopes, electron microscopes must work under the vacuum environment. Thus, they cannot be used to observe living biology samples. In addition, the electron beam can damage the illuminated specimens. Other issues (i.e., the optimal control of brightness of electron bean gun and the improvement of electron lens quality) still need investigations [14].

\subsection{Scanning tunnel microscopes}

The scanning tunnel microscope (STM) is also named "tunnel scanning microscope". This instrument detects the surface structure of the objects based on the tunnel effect of the quantum mechanics. STM applies a very thinning tip (in an atom unit of its head) to 
detect the sample surface. As the tip is very close to the sample surface $(<1 \mathrm{~nm})$, the atoms in the tip head overlap with the electronic cloud of electrons in the sample surface. If a biasing voltage is applied between the tip and the samples, electrons will pass through the barrier between the tip and sample to generate the tunnel current in the order of nano-amperes $\left(10^{-9} \mathrm{~A}\right)$. By controlling the distance between the tip and the sample surface and accurately moving the tip along the sample surface in the three-dimension, the detector can record the data related to the surface morphology, electronic surface states, and other relevant sample surface information [15]. The STM has the amazing ability to achieve the spatial resolution of less than $0.1 \mathrm{~nm}$ in the horizontal direction and less than $0.001 \mathrm{~nm}$ in vertical direction. Generally, when objects are in the solid state, the distance between atoms typically is from $0.001 \mathrm{~nm}$ to $0.1 \mathrm{~nm}$. Because of this, the STM enables scientists to locate the single atom and observe the status of the atoms and molecules in the conductive material and surface structure. Hence, the STM generates substantially higher spatial resolution than other similar atomic microscopes. On the other hand, STMs can use the tip of the probe needle to manipulate accurately the atoms under low temperature environment. This enables the STM to be used as both a measurement and a process tool in the nanometer technology [16-18]. One unique characteristic of STM is that, for the first time, one can observe the permutation status of the atoms in the object surface and the related physical chemistry characteristics of the electrons in the surface. Due to the width and scope of its perspectives in the research and application fields related to the surface, material, and life sciences, the invention of the STM was considered by the international scientific community as one of the ten most important achievements of the science and technology in 1980s.

In summary, increasing microscope resolution has been an actively pursued goal in the microscopic imaging field. Using these three types of microscopes, one may observe the biological cells and microorganisms using an optical microscope, observe virus using an electron microscope, and detect or visualize the atoms using a scanning tunneling microscope.

\subsection{Fluorescence microscopes}

Although the fluorescence microscope can be characterized as a branch of the optical microscopes, it has many unique image generation and application characteristics in the biomedical imaging research fields. This section discusses this in more detail. The fluorescence microscope [19] uses short-wave light to illuminate the examined specimen and make certain objects inside the specimen emit the fluorescent light. Based on the received fluorescent light, one is able to observe the shape and location of the target objects. Comparing the difference between the fluorescence and conventional optical microscopes, one of the major differences is that the fluorescence microscope uses the high-voltage mercury lamp to provide all band light illumination, using a projection method in which the light is projected to the specimen. Due to the typically weakly reflected fluorescent light, the microscope must have a large numerical aperture to allow the objects to be observed. As a result, the numerical aperture of the fluorescent microscopes is larger than conventional optical microscopes. The fluorescent microscopes also have a special filtering system, including the assembly of the illumination filters and cut-off filters. Specifically, the fluorescent microscope has the following unique characteristics or requirements:

1) It requires a light source that has sufficient power to emit fluorescent light;

2) It is equipped with a set of optical filters that fit the requirement of stimulating different objects to emit different fluorescent light. By selecting the suitable wavelength of the emitting light, the wavelength of the emitted light can coincide with that of the absorption light, resulting in the maximum fluorescent light output;

3) To acquire the weak fluorescent images, it is equipped with a set of cut-off light filters that allow only the selected fluorescent light to pass through the imaging system and block the other types of scattering light to increase the signal-tonoise ratio of the system; and

4) The system of optical magnification needs to fit the characteristic of the fluorescent light in order eventually to obtain the fluorescent images with the highest spatial resolution.

Due to these requirements, a modern fluorescent microscope typically is assembled based on the structure of a duplex optical microscope. The equipped fluorescent device includes fluorescent light source, emission light path, stimulation/emission light filters, and the other components. 
1) The fluorescent light source: It provides the light source that emits the light within the specific range of the wavelength or energy, which guarantees that the examined specimen acquires sufficient stimulation and generates strong fluorescent light. The fluorescent microscope typically uses the mercury lamp as the light source that is able to provide the exciting light with continuous wavelength. The mercury lamp has stronger energy in several commonly used light wavelengths (including $365 \mathrm{~nm}, 405 \mathrm{~nm}, 550 \mathrm{~nm}$, and $600 \mathrm{~nm}$ ).

2) An exciting light path: It serves as a fluorescent lighting device that contains a group of condenser lens and light focusing adjustment device. In the light path, the adjustable light and field apertures, (Neutral Density) ND filters, light splitter, and processing lens are installed.

3) Light filters: These are a set of the optical filtering components. Each has a certain wavelength width that selectively allows stimulated/emitted light pass through the optical system of the microscope.

Similar to the other conventional optical microscopes, the fluorescence microscopes can be divided into two categories based on their optical path [20]: transmission fluorescence microscopy (TFM) and epifluorescence microscopy. In TFM, the light passes through the condenser to excite the specimens to emit the fluorescence light. The TFM often uses the dark-field condenser that is able to adjust a reflector so that the light can illuminate the specimen from different directions. Compared to the old style fluorescent microscopes, the TFM has a number of advantages, which include producing strong fluorescence light when the specimen is observed in the low magnification. Disadvantages include the fact that as magnification levels increase, the fluorescence light weakens. Therefore, TFM is better used for observing larger specimens. As shown in Fig. 4, the light is emitted from one side of the specimen and the fluorescent light is collected by the lens in the other side of the specimen.

The recently developed epifluorescence microscopy is a new type of fluorescent microscopy [21]. The difference between the epifluorescence and the transmission fluorescence microscopy is that the objective lens in epifluorescence microscope serves first as a well-corrected condenser and then as the image-

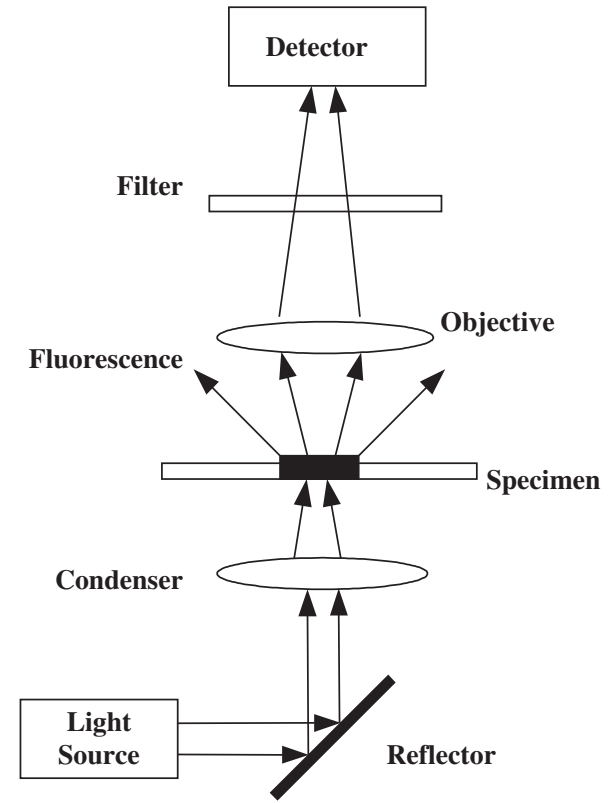

Fig. 4. Illustration of optical path of a transmission fluorescence microscopy.

forming light gatherer. The illuminator directs light onto the specimen by first passing the light through the microscope objective lens on the way toward the specimen and then using the same objective lens to capture the emitted light. The dichroic beam splitter is placed between the optical path titled at $45^{\circ}$, and the reflected excitation light passes through the objective lens and eventually reaches the specimen. The fluorescent light emitted by the specimen, as well as the reflected exciting or scattering lights, passes through the objective lens simultaneously and arrives at the dichroic separator (mirror) that separates the fluorescent light and the original exciting light. The remaining scattering light is absorbed further by the cut-off filter. The advantages of the epifluorescence microscope include the uniform illumination, sharp image, and the strong fluorescent light as the increase of the magnification level.

The optical path of an epifluorescence microscope is illustrated in Fig. 5. After the reflection from the dichroic mirror, the excitation light converges at the specimen. The emitted fluorescent light from the specimen first passes through the objective lens and the dichroic mirror, and then is collected by detection sensor (probes). The key component of an epifluorescence microscope is the dichroic beam splitter. It includes a multilayer optical interference filter that is installed 


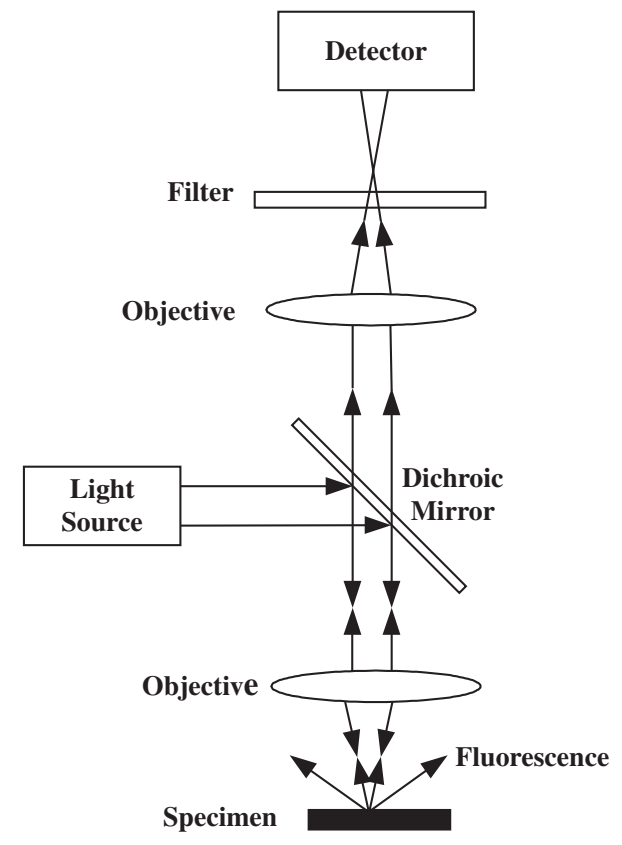

Fig. 5. Illustration of the optical path of a typical epifluorescence microscopy.

with a titled angle of $45^{\circ}$ along the optical path. It is able selectively to reflect or transmit the light only in certain wavelength range.

Although the transmission fluorescence microscopes can yield images with sufficiently high contrast in low magnification, the image is often relatively dark compared to the use of the epifluorescence microscope. Thus, the epifluorescence microscope is more popular in clinical practice to date. In addition, compared to the transmission fluorescence microscope, the epifluorescence microscope has following characteristics.

1) The microscope objective lens also serves as a light condenser. Thus, the alignment issue of the condenser is avoided. In particular, the fluorescent light intensity of the image increases as the increase of the numerical aperture (NA) of the object.

2) Since excitation light does not pass through slides in epifluorescence microscopy, it reduces the light loss. Meanwhile, the excitation and fluorescent light travel in the opposite direction to the objective lens. Two light beams separate with each other without interference. Some scattered excitation light may reach the object, and they are mostly reflected by the dichroic mirror to the light source. Thus, a very thin filter typically is installed between the objective lens and the detector (probe) to absorb a small fraction of remaining scattering light passing through the objective lens.

3) Unlike the use of transmission-type of excitation, in which most of fluorescence light creates in the bottom section of the specimen and must transmit through the specimen slice producing unavoidable scatter light, in epifluorescence microscope, the light source and the observation plane locate in the same plane. Therefore, there is no loss of the fluorescence intensity and no effect on image quality. In particular, it has unique advantages when it is used to examine the thick specimen slices with bacteria and tissue culture.

4) Since the ultraviolet (UV) light needs to pass through the objective lens, the transmission coefficient of the objective lens must be considered. As a result, a series of special objective lenses that can transmit UV light have been designed and manufactured. However, such a special objective lens is not required in the transmission fluorescence microscope.

\section{The application of microscopes in pathological imaging}

The invention of the microscope has significantly promoted the development of biology and medicine. It demonstrates the mystery of the microscopic world and reveals the causes of disease from the microscopic view. By acquiring the microscopic and pathologic images of the pathogenic microorganisms, medical researchers have established a series of new academic subjects, including bacteriology, immunology, virology, cell pathology, and others. In particular, the invention of electron microscopes has raised the observation of the traditional pathology from the cellular level to the sub-cellular level. As a result, one cannot practice modern medicine without microscopes. New microscopes such as the confocal laser scanning microscope (CLSM) [22], atomic force microscope (AFM), and scanning tunneling microscopy (STM) further improve the resolution of pathology imaging [23]. In this section, we briefly discuss the application of microscopes in assisting the clinicians in biomedical engineering, particularly in diagnosing pathological images. 


\subsection{Optical microscopes}

Optical microscopes have been used widely in the pathology related clinical laboratories to diagnose a variety of diseases based on the examination of the body fluid change, invaded virus, and the variation of atomic structures [24]. Such diagnostic information and reports provide the clinicians with valuable references to select and implement the optimal treatment plans for the patients and monitor their efficacy. In genetic engineering and microscopic surgery, microscopes are essential tools for clinicians. Although optical microscopes are still considered to be very important tools in the clinical facilities, the development of the originally optical microscopes is quite mature to date; in particular, the optical characteristics of the microscopes have reached perfect degree without much space left to make further improvement. Therefore, the current development of the optical microscopes focuses on finding new application fields, making the systems simple and easy to use, as well as making one system for the multiple applications.

Since the biological specimen is typically one kind of muddy medium, it can generate the strong light scattering and absorption. As a result, the light (or optical wave) cannot penetrate deeply into the interior of the biological structures; thus, it is unable to extract a clear image of the biological structures. With the rapid advance of photonics, new imaging methods based on photonics have been developed, including the laser confocal scanning microscope and the near-field optical scanning microscop $[25,26]$. First, the laser confocal scanning microscope is the combination of laser source and the confocal microscope. Specifically, it is a system that uses both the confocal concept of the traditional optical microscopy and the laser as the light source. It then uses the computer to process and analyze the acquired digital images of the observed specimens. By continuously scanning the multiple layers of the living cells or tissue slices, the laser confocal scanning microscope can acquire the complete $3 \mathrm{D}$ images of each structural layer of a single cell or local structure of a group of cells. Based on the near-field probing theory, the near-field optical scanning microscopy is a new technology developed by using the optical scanning probe needles. It breaks through the limitation of optical diffraction and is able to reach the spatial resolution in the range from $10 \mathrm{~nm}$ to $200 \mathrm{~nm}$. By further combining with the related optical spectroscopy, using the near-field optical scanning microscopy creates a new approach to detecting and examining spectrum images of small biological specimen beyond nanometer range. As a result, researchers are able to analyze the single biological atom to date.

\subsection{Electron microscopes}

There are number of differences between electronic and optical microscopes when applied to pathological images. When using optical microscopes to observe pathological specimens, the observer routinely needs to adjust the micro-focusing system of the microscope and move the specimen to achieve the optimal observation of the tissue structure of the specimen. Using optical microscopes also generates less eye fatigue due to small light simulation to the eyes. However, the magnification power of the optical microscope is much lower than that of the electronic microscope. In addition to the higher magnification power, an electronic microscope can display more detailed tissue structures on the monitor (screen). It also may be able to conduct secondary magnification of the specimen displayed on the screen. Thus, the electronic microscope plays an important role in studying human organ tissue structures and the other sub-nanometer structures.

Due to the short wavelength of the electron beams, the electrical microscopes break through the limitation of the spatial resolution using optical microscopes, which opened a new door for the human eyes to observe the small structures at the molecule and/or atomic level. For example, using electronic microscopes to observe cells, researchers clearly have confirmed the existence of a cell membrane that consists of three thin layers with equal thickness but different density. Two external layers have higher density than the middle (interior) layer. When using electronic microscopes to observe unmyelinated nerve fiber, it was found that the nerve membrane cell of the fiber contained multiple shafts (i.e., 2 to 9). When using electronic microscopes to observe and study muscle structure, researchers discovered two important characteristics of muscle fiber: (1) the muscle fiber depicts the horizontally bright and dark stripes in the regular arrangement (namely the structure of horizontal stripes), and (2) muscle fiber is actually formed by finer fiber units that are perpendicular to the main fiber. Using electronic microscopes to study atomic structure of nucleic acid, observers can identify the linear status inside the nucleic acid atom structure with diameter around $20 \mathrm{~A}^{\circ}$. These examples indicate that the application of electronic microscopes 
significantly expedites the possibility of exploring the secret of life and also plays an important role in the advance of medicine, as well as the improvement of human health [27].

In pathology, electronic microscopes have showed their importance in many applications. For example, electronic microscopes have been applied to pathological diagnosis of renal biopsy specimens. Renal glomerular disorder is a commonly diagnosed disease in clinical practice and can be classified as primary and secondary types of disorders. In order to more accurately diagnose the pathology of renal glomerular disorder, renal needle biopsy often is used. Besides distinguishing the different patterns of the morphological and tissue elements inside glomeruli, electronic microscopes can be used to observe and detect microstructural aberration or changes, especially in epithelial cells, mesenterium, base membrane, and interstitial substance, which cannot be observed or detected using the conventional optical microscopes. By analyzing the observed micro-changes, physicians can find whether there are electron-dense deposits and corresponding locations. However, the single electronic microscopic technology also has limitation. In order to make renal needle biopsy more useful and accurate in the pathology diagnosis, the combination of multiple technologies, including the optimal use of conventional optical microscopes, immune-fluorescence microscopes, and electronic microscopes, is needed [23].

Using the electronic microscope not only has improved and enhanced the knowledge of the pathology, but also has provided a reliable foundation for the diagnosis of many diseases. With the rapid advance of science and technology, electronic microscopes have been and will continue be extensively used in pathologic diagnosis. However, single electronic microscopic technology has some limitations in pathologic diagnosis. For example, the field of vision (FOV) is reduced substantially due to the high spatial resolution of the electron microscope. Each FOV typically can only show a single cell or the partial structure of the cell. Meanwhile, observers can only observe still and dead specimens instead of the cells in vivo. Therefore, while electronic microscopes have been used actively in clinical practice, one also needs to combine other technologies such as the quantitative analysis of the morphology of the micro-structural changes using image processing, the nucleic acid hybridization in situ technology, and the apoptotic indices of the observation using electronic microscopes. Mean- while, the scanning tunnel microscope and the atomic force microscope also have been introduced recently into the pathological area to improve the electronic microscopic technology. With closer connections to computer technology, the observers (i.e., pathologists) more easily and conveniently can use and control electronic microscopes through the connected computer and networking systems.

\subsection{Fluorescence microscopes}

Irradiated by the short wavelengths of light (e.g., ultraviolet and violet blue light $250 \mathrm{~nm}-400 \mathrm{~nm}$ ), some substances can be excited by absorbing the energy and emit a longer wavelengths of light with energy degradation, which includes blue, green, yellow, or red light within the wavelength between $400 \mathrm{~nm}$ and $800 \mathrm{~nm}$. This is called photoluminescence [28]. Some specimens naturally can generate fluorescent light when they are illuminated by the light with the proper wavelength. For example, most lipid and protein can emit fluorescent light in blue. This phenomenon is called auto fluorescence or primary fluorescence. However, most substances generate fluorescence light only when they are dyed with fluorochromes and are exposed in the light of short wavelengths.

In the 1930s, florescent staining was applied to investigating and observing the morphology of bacteria, mildew, cells, and fiber. For example, Mycobacterium tuberculosis can be detected in sputum by acid- fast bacteria staining methods. In the 1940s, the fluorescent staining protein technology was invented and applied extensively to the routine clinical immunofluorescence staining, which can examine and locate virus, bacteria, mildew, protozoan, parasite, antigen, and antibody in animals and humans. It has been used to investigate the pathogenesis and the cause of diseases such as categorizing and diagnosing glomerular diseases and identifying the relation between human papillomavirus (HPV) and the cervical cancer. The fluorescence microscope, a special tool for detecting fluorescence, has been getting more and more applications in the clinical research and disease diagnosis [29]. The coupling system between a fluorescence microscope and a fluorescence spectrometer has been extensively used in cell biology, biochemistry, physiology, neurobiology, and pathology. These systems can provide qualitative and quantitative analysis for various structures in either living and fixed cells or tissues. Fluorescence microscopes can observe cells and 
structures either emitting auto fluorescent or stained by the fluorochromes. The light source of a fluorescent microscope is a high-voltage mercury lamp that emits the ultraviolet light with short wavelengths. The fluorescent microscope also is installed with excitation and dichroic filters. The fluorochromes in the specimens are excited by the ultraviolet light and emit light with different colors. This enables one to analyze the distribution and locations of fluorescence inside the cells or structures. Some tissues can emit auto or spontaneous fluorescent light. For example, lipofoscin inside nerve or myocardial cells emit brown fluorescent light; Vitamin A of epithelia cells in hepatic Ito and retinal pigment emit green fluorescent light; and monoamine (e.g., Catecholamine, 5 - HT, Histamine) inside neuroendocrine cells and nerve fibers emits light with different colors under formaldehyde. Quinine and Tetracycline inside the tissues also emit fluorescent light. Some tissues inside the cells can bind with fluorescein to emit fluorescent light. For example, Ethidium bromide and Acridine orange can combine with DNA to measure the content of DNA. The fluorescence microscope also has been widely used in the immunocytochemistry research. By using fluorochrome to mark Isothiocyanate and Rhodamine, the marked antibody directly or indirectly can bind with the corresponding antigen. Thus, clinicians can detect the existence and distribution of antigen.

Since the invention of first fluorescence microscope in 1908, fluorescence detection has been improving constantly. This includes the introduction of highvoltage mercury lamps and the development of the multi-layer coating technology that is able to manufacture the high quality excitation and cut-off filters. With high quality optical components, the fluorescent microscope made a great contribution to the development of immune-fluorescence in the 1980s. In the 1990s, the laser scanning confocal fluorescence microscopy was developed. It measures and determines the fluorescence distribution within cells and tissue biopsies and obtains real-time overlapping phase-contrast and fluorescence images, as well as the primary color images labeled with double fluorescents. Currently, more advanced technologies related to the single-photon fluorescence microscopy, two-photon fluorescence microscopy, 4 pi confocal fluorescence microscopy [30], and full internal reflection fluorescence microscopy [31] (total internal reflectance fluorescence microscopy) have been developed. As a result, using these new tools, the technology and application of fluorescence detection can be applied or implemented in more research and clinical applications in pathology and other biomedical fields.

\section{Digital microscope imaging}

Although microscopy plays a very important role in biology and medicine, the observation is performed with the naked eye under the conventional optical microscopes. This can result in eye fatigue after a relatively long time of continuous observation. In addition, the image information cannot be stored and processed for different image enhancement purposes. To solve these limitations, digital microscopes have been developed and tested. A digital microscope (or imaging system) is an integrated design that combines traditional optical microscope, digital multimedia, and digital processing technology [32, 33]. As shown in Fig. 6, a digital microscope imaging system typically includes three components: Microscopy optical module, data acquisition module, digital image processing, and software control modules. Among these modules, the optical module realizes the function of the microscopic imaging; data acquisition module records the images produced by digital video devices, including CMOS, CCD, digital camera stored in optical module in the digital format, and then transfers these digital images to the computer storage devices through different graphics card interface or USB interface; and the software control module, the core of the whole system, controls the image capture, processing, and measurement in real-time to optimally improve the image quality. The digital images can be monitored in real-time using a color TV or computer monitors. After using a variety of digital image processing methods, digital microscopic imaging systems more sensitively can capture and display the image details. In fact, installation of online image acquisition, processing, and analysis systems have become an important symbol of modern advanced microscopes.

Due to its significantly technological advantages, microscopic digital imaging technology also has been used and/or integrated in a variety of electronic microscopes. For example, unlike the traditional electron microscopy imaging that uses electronic sensitive floor to generate photographic images, the digital microscope equipped with the CCD imaging system converts electrons that carry image information through electro- 


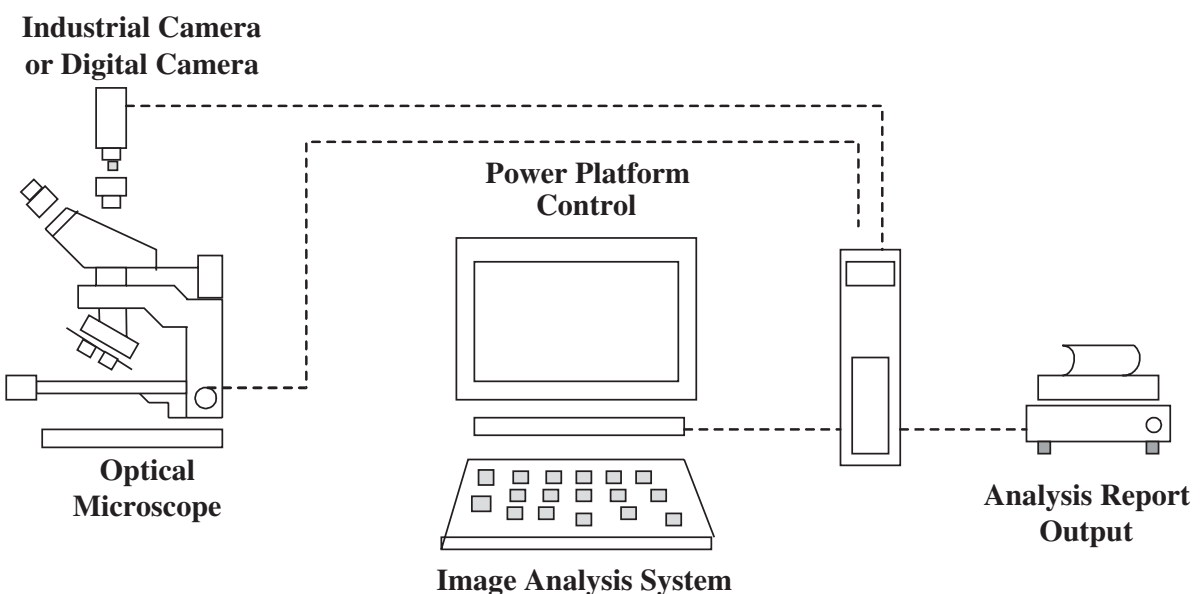

Fig. 6. The configuration of modern digital microscope imaging system.
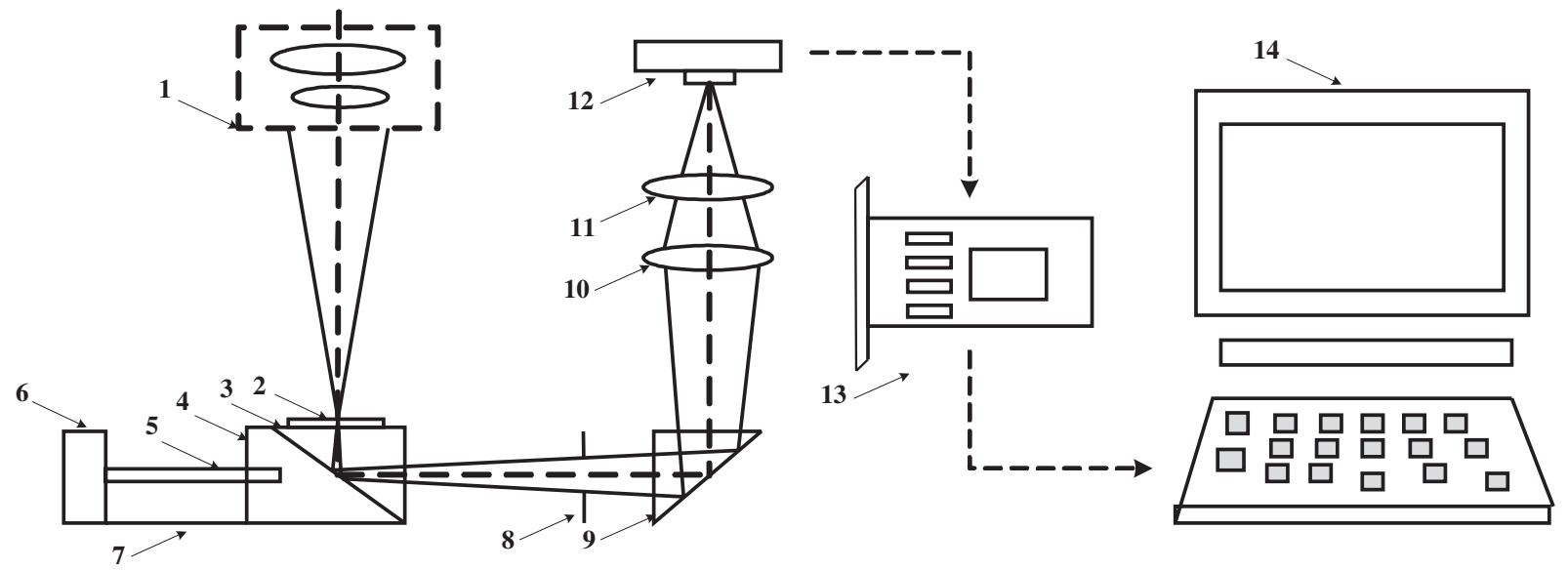

Fig. 7. The working principle of digital microscopic imaging system. 1 - Electron microscope lens; 2 - Fluorescence plate; 3 - Prism; 4 - Prism Block; 5 - Drive screw; 6 - Motor; 7 - Base; 8 - Aperture; 9 - Prism; 10 - Lens; 11 - Lens; 12 - CCD; 13 - Image acquisition card; and 14 Computer.

optical conversion devices to the light signal and sends the signal to the CCD. After the images are captured with CCD, they can be sent to the computer through an image acquisition card to perform a variety of required image processing procedures (as shown in Fig. 7).

Thus, digital microscopic imaging technology is an extension of conventional microscopy. It integrates the microscopy, image acquisition, and computer control and processing technology to manage and control the whole imaging process, including image acquisition, sampling, processing, and data storage. Among these, the image acquisition and processing technology is the core for digital microscopic imaging technology.

Current digital image acquisition devices can be divided into three categories, including the use of (1) analog cameras plus video capture card, (2) consumer grade digital cameras, and (3) professional grade digital cameras. These three devices have their own characteristics and application fields in which professional grade digital cameras are the most popular choice in digital imaging microscopy systems. The introduction of digital imaging technology creates a great opportunity for the post-processing of acquired images. In particular, with the rapid advance of more powerful computers, digital microscopic images can be more effectively and efficiently processed and analyzed.

Image analysis and processing may be customdesigned or may use sophisticated commercial microscopic image processing software packages. Many 
professional software packages are well designed with variety of useful functions or schemes. They can provide both simple geometric measurement and sophisticated analysis to identify the relationship between the complex geometric structures. By providing the absolute space calibration to ensure the most accurate measurements and the advanced image segmenting technology, these commercial schemes can help observers to better distinguish the overlapping objects, detect the contours and shapes of the small objects, identify similar objects or groups, and categorize or mark the different objects with different colors. Some schemes can be used as advanced analytical tools that use the spectrum diagram, spectral profile, pseudo-color, three-dimensional surface shape, and other methods to manipulate the data and display it in different formats.

The advantage of connecting the microscope to a computer is that it produces digital images. Using these digital images, the research scientists and clinicians can apply various image processing and analysis software to obtain needed experimental data for variety of purposes. For example, using digital microscopes, one can replace the manual process of counting red tide organisms, a very tedious and time-consuming task. The computerized program is able to count the total number of red tide organisms depicted in a sample and classify red tide organisms in different size groups. The magnified red tide organisms can be displayed on the computer screen. As a result, by combining with manual identification, this computerized method greatly reduces the labor intensity of manual count using a conventional optical microscope.

\section{New development in microscopic imaging technology}

With the advance of technology and the special requirements of research projects, many new microscopic imaging methods have been developed and tested that aim to acquire the images with high resolution and large field of depth, as well as in threedimensional stereoscopic format. As a result, using these new microscopic imaging systems and acquired images, the clinicians are able to conduct dynamic, non-destructive, and non-intervention in vivo (real time) tests in the biomedical research and clinical practice. Following are several new developments in this field.

\subsection{Coexistence of a large field depth and high magnification power}

In the conventional optical microscope, a large depth of field cannot coexist with high magnification power. As a result, due to the limited depth of field, the optical microscope cannot capture clear images of the targeted small objects located at different depths of the sample surface. The use of a scanning electron microscopy (SEM) or a confocal microscopy can resolve the contradiction between magnification and depth of field. As a result, one can observe clear (sharp) images with larger depth of field.

The SEM uses a very thin electronic beam to scan the sample surface. Electrons produced by scanning are collected by a specially designed detector. The corresponding electrical signals are further delivered to the CRT monitor screen to generate the three-dimensional image of the sample surface. These images also can be captured into the pictures. The resolution of the SEM lies between optical microscopy and transmission electron microscopy (TEM) (i.e., up to $3 \mathrm{~nm}$ ). Meanwhile, the depth of field of the SEM is a hundred times higher than the optical microscope and at least ten times greater than the TEM. Thus, it is able to acquire a clear picture with a large depth of field.

The confocal microscope is an integrated technology that combines the optical and digital image analysis technique. It is a new microscopic imaging technology specifically useful in acquiring the images of non-planar samples. It can obtain enormous depth of field and exquisite details and also can acquire true-color information that often cannot be obtained using an electron microscope. When using the confocal microscope, one first adjusts the focus to search for and reach the samples distributed in different depth levels, captures all the images distributed in these levels with digital imaging devices, and transfers them to a computer (each of these aspects of the image has a clear location different from other images that record different parts of sample morphology and color information). After data analysis and integration using the special image processing software, the final high quality and clear picture is produced.

\subsection{Three-dimensional imaging}

The modern microscopy is not only limited to observing the specimen surface. By combining laser and confocal microscopy, researchers and clinicians 
can acquire and view the images of the cross sections of specimens at different depth levels. Then, using computerized image processing and $3 \mathrm{D}$ reconstruction algorithms, the three-dimensional shape profile of specimens can be acquired in high-resolution.

The main principle of laser scanning confocal microscope (LSCM) is to let the laser beam pass through a pinhole to generate a point light source. The laser light passes through the excitation filter and arrives at the beam splitter. Because the beam splitter can reflect excitation light with a shorter wavelength and transmit the light with a longer wavelength, the excitation light is reflected at the beam splitter and transmits through the objective lens. The laser beam scans different focal planes marked by the fluorescent agents under the control of the scanning control device. The excited emission light from the fluorescent marks passes through the original incident light path and directly returns to the beam splitter. After passing through the transmission filter for the specific wavelength, the emission light finally reaches the detection pinhole of the photomultiplier tube (PMT). By converting the PMT current into the digital signals, an image is created and displayed on the computer monitor screen.

Since LSCM can continuously scan the living cells and tissue or cell biopsy samples layer by layer to obtain images at different depth levels, it is also named as "non-destructive optical biopsy". The distance between two LSCM scanned layers can be less than $0.1 \mathrm{um}$. Using computerized reconstruction algorithms, one can generate or acquire three-dimensional images that are able to observe or access the sophisticated cytoskeleton, chromosomes, organelles, and cell membrane.

\subsection{In vivo detection}

Observing and monitoring living cells can be very useful in many research applications, but it is a difficult technical challenge in the microscopic imaging field. To enable researchers or clinicians to observe the detailed structures of the specimens and also dynamically monitor the function of living cells or tissues in real-time using microscopic pathology images, several new technologies have been developed and tested. For example, by applying the specific fluorescence probes to label the material to be observed, the researchers can use LSCM to monitor dynamically the whole process of change in different locations or tissues of the cells after receiving stimulation in real time. LSCM also can be used in many applications such as assaying the changes within the $\mathrm{PH}$ of the cells, detect potential changes in membranes, detecting the production of intracellular reactive oxygen, testing the process of drugs into the tissue or cell membrane and locating its position, measuring the fluorescence resonance energy transfer (FRET), and real-time monitoring many other living cells.

\section{Summary}

Pathological imaging technology is a fast growing area of academic investigations, industrial developments and clinical applications. This paper presents a brief review of the fundamental concepts and a discussion on the recent developments and clinical potential of contemporary microscopic imaging techniques. The conventional optical microscopy has been an important tool in clinical pathology. The interface of optical microscopy with digital image acquisition methods combines the power of optical imaging, electronic detection, and computerized analysis. These and other emerging technologies will continue to evolve, enable cellular-, molecular-, and genetic-imaging, as well as tissue imaging with high efficiency and accuracy, to facilitate clinical screening and diagnosis.

\section{Acknowledgements}

The authors would like to acknowledge the support in part of the Charles and Jean Smith Chair Endowment fund, and the support in part of NIH grant RO1CA136700.

\section{References}

[1] W.J. Croft, Under the Microscope: A Brief History of Microscopy, World Scientific Publishing, Singapore, 2006.

[2] H. Bethge, Electron microscopy-beginnings and present, Ultramicroscopy 10(3) (1982), 181-186.

[3] A. Bogner, P.H. Jouneau, G. Thollet, D. Basset and C. Gauthier, A history of scanning electron microscopy developments: Towards "wet-STEM" imaging 38 (2007), 390-401.

[4] G. Binnig and H. Rohrer, Scanning tunneling microscopy, Helvetica Physica Acta 55(6) (1982), 726-735.

[5] L.C. Martin, The Theory of the Microscope, Blackie, 1966.

[6] S. Inoue and K.R. Spring, Video Microscopy: The Fundamentals, Plenum Press, New York, 1997.

[7] M. Bass, Handbook of optics, Geometrical and Physical Optics, Polarized Light, Components and Instruments, Volume 1, 3rd edn, McGraw-Hill Companies, New York, 2010. 
[8] J.S. Park, C.K. Choi and K.D. Kihm, Optically sliced microPIV using confocal laser scanning microscopy, Experiment in Fluids 37(1) (2004), 105-119.

[9] G.R. Dickersin, Diagnostic Electron Microscopy, Igaku-Shoin Medical Publishers, New York, 1998.

[10] M. Abramowitz, Microscope Basics and Beyond, Olympus Corporation Publishing, New York, 1987a.

[11] M. Abramowitz, Contrast Method in Microscopy: Transmitted Light, Olympus Corporation Publishing, New York, 1987b.

[12] M. Abramowitz, Fluorescence Microscopy: The Essentials, Olympus Corporation Publishing, New York, 1987c.

[13] M.W. Davidson and M. Abramowitz, Optical Microscopy, Olympus America, New York, 1999.

[14] T.D. Allen, Introduction to electron microscopy for biologists, in: Methods in Cell Biology, Volume 88, Academic Press, London, 2008.

[15] J.A. Stroscio and W.J. Kaiser, Scanning tunneling microscopy, in: Methods of Experiment Physics, Volume 73, Academic Press, London, 1993.

[16] M.H. Weinstein and J.I. Epstein, Telepathology diagnosis of prostrate needle biopsies, Hum Pathol 28(1) (1997), 22-29.

[17] Y. Ando, Development of three-dimensional electrostatic stages for scanning probe microscope, Sensors and Actuators A 114 (2004), 285-291.

[18] S. St Fahlbusch, J. Mazerolle, M. Breguet, et al., Nanomanipulation in a scanning electron microscope, Journal of Materials Processing Technology 167 (2005), 371-382.

[19] B. Herman, Fluorescence Microscopy, World Publishing Corporation, Beijing, 1998.

[20] Y. Guanglie, Fluorescence and fluorescence microscope, Optical Instruments 23 (2001), 18-29.

[21] J. Slavik, Fluorescence Microscopy and Fluorescent Probes, Plenum Press, New York, 1997.
[22] G.S. Kino and T.R. Corle, Confocal Scanning Optical Microscopy And Related Imaging Systems, Academic Press, London, 1996.

[23] E.C. Ho, L.J. Baker, H. Savage, et al., Teaching arms and screens for microscope and fibreoptic nasendoscope: Are they effective teaching devices and are we utilizing them? Eur Arch Otorhinolaryngol 267 (2010), 643-645.

[24] A.J. Lacey, Light Microscopy in Biology - A Practical Approach, 2nd edn, Oxford University Press, Oxford, 1999.

[25] J.B. Pawley, Handbook of Biological Confocal Microscopy, 3rd edn, Springer, New York, 2006.

[26] M.A. Pawslear and P. Moyer, Near Field Optics: Therapy, Instrumentation, and Applications, Wiley, New York, 1996.

[27] A. Okumura, J. Suzuki, I. Furukawa, et al., Signal analysis and compression performance evaluation of pathological microscopic images, IEEE Trans Med Imaging 16 (1997), 701-710.

[28] D.A. McQuarrie and J.D. Simon, Physical Chemistry, A Molecular Approach, University Science Books, Sausalito, 1997.

[29] G. Cox, Optical Imaging Techniques in Cell Biology, CRC Press, Boca Raton, 2007.

[30] S. Hell and E. Stelzer, Properties of a 4 Pi confocal fluorescence microscope, Optical Society of America 9 (1992), 2159-2166.

[31] D.J. Arndt-Jovin, M. Robert-Nicoud, S.J. Kaufman, et al., Fluorescence digital imaging microscopy in cell biology, Science 230 (1985), 247-256.

[32] P. Gualtieri and P. Coltelli, A digital microscope for real time detection of moving microorganisms, Micron and Microscopica Acta 20 (1989), 99-105.

[33] S. Tucker, W.T. Cathey and E. Dowski, Extended depth of field and aberration control for inexpensive digital microscope systems, Optics Express 4 (1999), 467-474. 


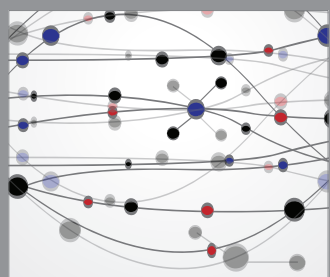

The Scientific World Journal
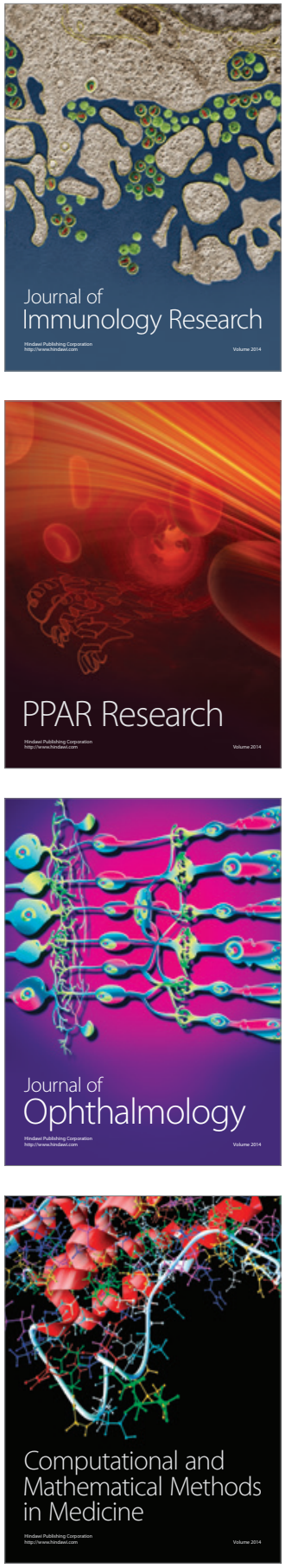

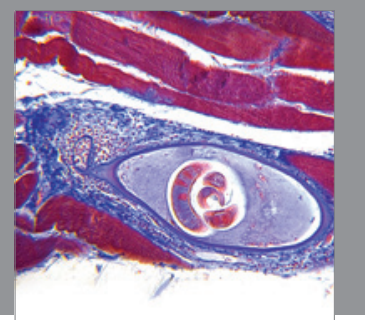

Gastroenterology

Research and Practice
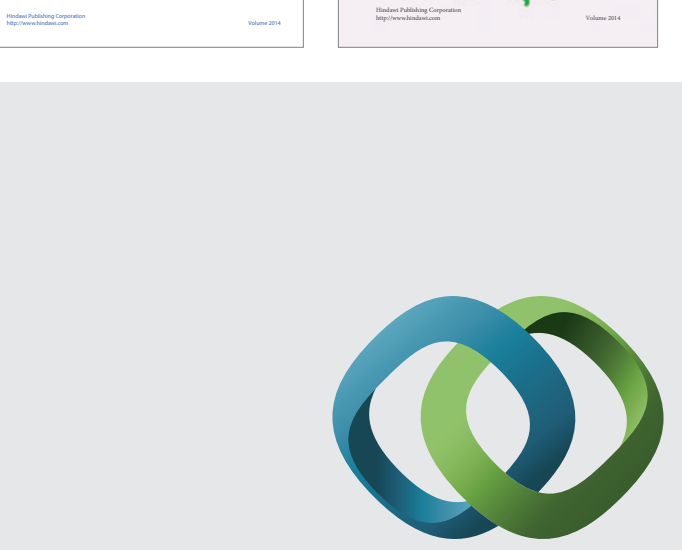

\section{Hindawi}

Submit your manuscripts at

http://www.hindawi.com
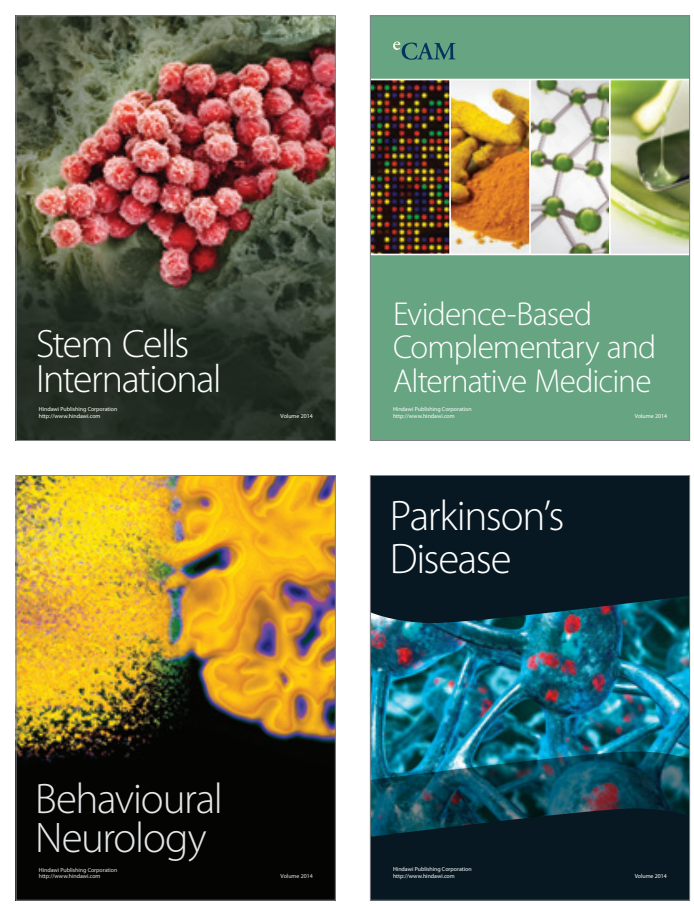

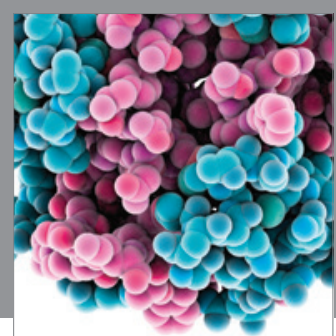

Journal of
Diabetes Research

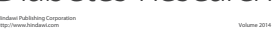

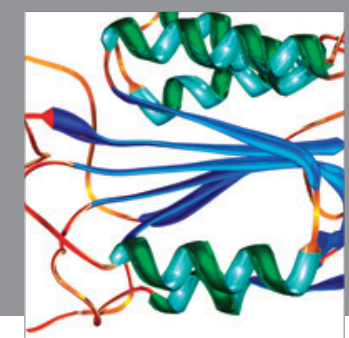

Disease Markers
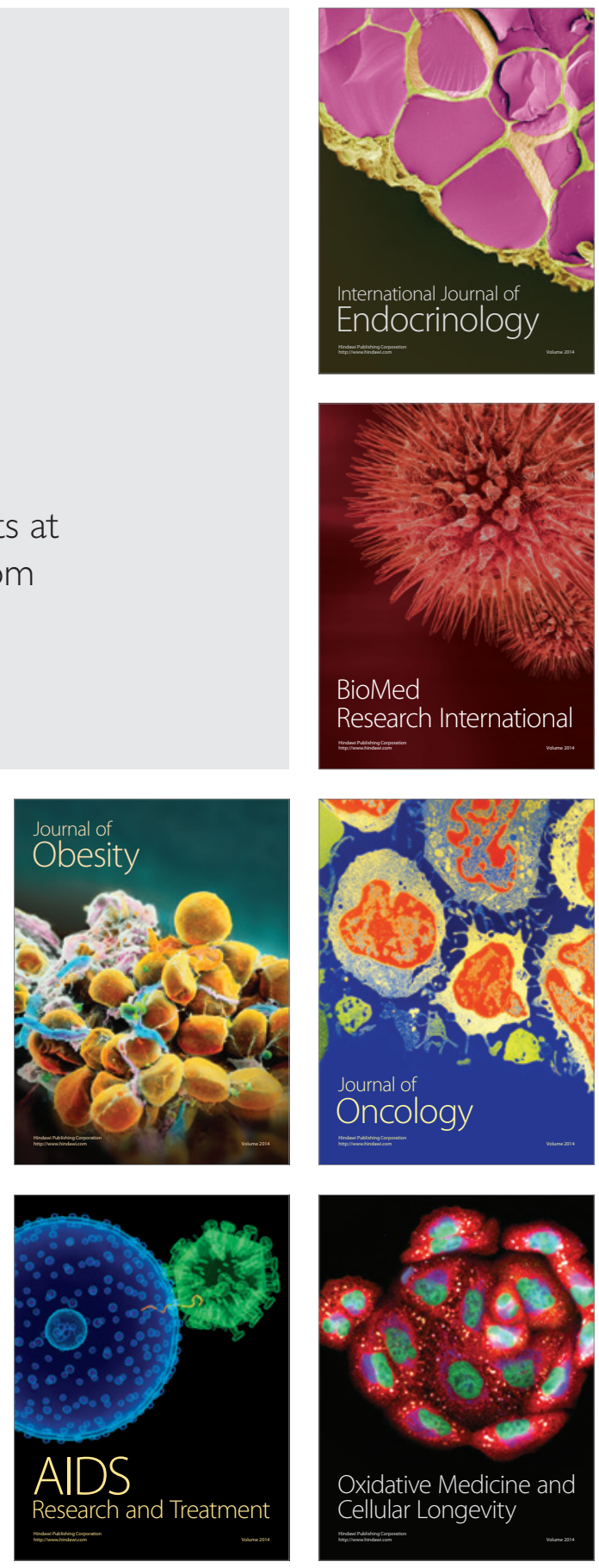